\title{
INFLUÊNCIA DA PINEAL SOBRE A MORFOLOGIA DO CÓRTEX DA ADRENAL
}

\author{
PINEAL 'S INFLUENCE UPON THE ADRENAL CORTEX \\ Lilian Margareth B de Lima'; Marcus Aurelho de Lima²; Cláudia Valéria C Rita33; Paulo Fernando de Oliveira4; \\ Luis Carlos dos Reis ${ }^{5}$; Maria de Fátima Borges ${ }^{6} \&$ Gilberto Pereira da Silva ${ }^{7}$ \\ ${ }^{1}$ Docente da Disciplina de Patologia Geral da Universidade de Uberaba; ${ }^{2}$ Docente da Disciplina de Endocrinologia da Faculdade de \\ Medicina do Triângulo Mineiro (FMTM); ${ }^{3 e}{ }^{4}$ Acadêmicos de Medicina da FMTM; ${ }^{5}$ Docente da Disciplina de Fisiologia da FMTM; ${ }^{6}$ Docente \\ da Disciplina de Endocrinologia da FMTM; ${ }^{7}$ Docente da Disciplina de Bioestatística da FMTM. \\ CorRespondênCIA: Dr. Marcus Aurelho de Lima - Disciplina de Endocrinologia - Hospital Escola - FMTM- Rua Getúlio Guaritá n 130 \\ CEP 38025 - 440, Bairro Abadia, Uberaba - MG. Fone: 034-318 5292/5258; Fax: 034-312 6640. E-mail: lima@mednet.com.br.
}

LIMA LMB; LIMA MA; RITA CVC; OLIVEIRA PF; REIS LC; BORGES MF \& SILVA GP. Influência da pineal sobre a morfologia do córtex da adrenal. Medicina, Ribeirão Preto, 35: 36-40, jan./mar. 2002.

RESUMO: Várias glândulas interferem na produção e secreção dos hormônios corticais da adrenal e, como previamente demonstrado por diversos estudos, a pineal exerce efeito inibitório sobre tais hormônios. A pinealectomia aumenta o nível dos hormônios do córtex e promove modificações morfológicas no córtex, tais como, diminuição da atividade mitótica e hipertrofia da cortical. A finalidade do presente estudo foi verificar a influência da pinealectomia, em ratos, sobre a morfologia das glândulas adrenais. Foram selecionados 4 grupos de animais, um grupo-controle normal, um submetido a cirurgia ficticial, dois pinealectomizados, sacrificados em intervalos de tempo diferentes. Realizou-se análise morfométrica e da proliferação celular das camadas corticais das adrenais. O estudo morfométrico demonstrou aumento da camada fasciculada dos animais pinealectomizados, em relação às adrenais dos ratos não pinealectomizados. Não foi observada proliferação celular em qualquer dos grupos estudados. Os resultados demonstram que a pineal exerce influência sobre as adrenais e a ausência desta glândula causa aumento do peso da adrenal por hipertrofia celular da zona fasciculada e não por hiperplasia.

UNITERMOS: Corpo Pineal. Córtex Supra Renal. Divisão Celular. Hipertrofia. Morfologia.

\section{INTRODUÇÃO}

As adrenais são glândulas endócrinas, que produzem mineralocorticóides, glicorticóides e esteróides sexuais pelo córtex e catecolaminas pela medula ${ }^{(1)}$.

A secreção dos citados hormônios é influenciada por algumas glândulas, tais como a hipófise, através do hormônio adrenocorticotrófico, estimulando o crescimento de zonas específicas do córtex da adrenal; a tiróide, por aumentar a taxa de inativação dos glicocorticóides no fígado, diminui sua concentração no sangue. $\mathrm{O}$ estradiol, secretado pelos ovários, parece também estar relacionado à produção dos hormônios do córtex da adrenal ${ }^{(2)}$. Além disso, estudos têm demonstrado a influência inibitória da glândula pineal sobre o córtex da adrenal ${ }^{(3 / 8)}$.

Alguns autores sugerem que a pinealectomia exerceria um efeito inibitório sobre a resposta inflamatória ${ }^{(9)} \mathrm{e}$ a glândula pineal, através de interação com circuito envolvendo a área septal, hipotálamo e amígdalas, teria efeito direto sobre o consumo de água e $\mathrm{sal}^{(10)}$. Ainda, a ausência da pineal aumentaria a atividade da 5-alfa-redutase ${ }^{(11)}$ e do nível de glicocorticóides e mineralocorticóides em ratos hipertensos ${ }^{(3)}$. O 
aumento dos hormônios corticais da adrenal, decorrente da pinealectomia, é abolido após a hipofisecto$\mathrm{mia}^{(12)}$. Além disso, há trabalhos, ressaltando que a atividade mitótica das células da medula da adrenal pode ser alterada pela pineal, de acordo com a fase do dia, diminuindo durante o escuro ${ }^{(8)}$.

Muitos estudos observaram modificações na morfologia da adrenal após pinealectomia, tais como aumento do peso ${ }^{(5,6)}$ e diminuição da atividade mitótica das células do córtex da adrenal(7). Vaughan et al., $1972^{(13)}$ demonstraram que a hipertrofia adrenal, decorrente da pinealectomia e/ou da adrenalectomia unilateral, em ratos, pode ser bloqueada pela administração de melatonina.

Diante do exposto, propõe-se verificar a influência da pinealectomia, em ratos, sobre a morfologia das glândulas adrenais, através de estudos morfométricos e de proliferação celular no córtex.

\section{MATERIAL E MÉTODOS}

\subsection{Seleção da Amostra e Grupos Experimen- tais}

Neste estudo, foram utilizados ratos (Rattus norvegiccus), machos, da linhagem Wistar, com peso corporal entre 200 a $300 \mathrm{~g}$, distribuídos em quatro grupos de dez animais cada um, sendo dois grupos-controle, $\mathrm{N}$ (controle normal) e CF (submetidos à cirurgia fictícia) e dois grupos experimentais, $\mathrm{P}_{1}$ (pinealectomizados e avaliados entre 15 e 25 dias após a cirurgia) e $\mathrm{P}_{2}$ (pinealectomizados e avaliados entre 60 e 70 dias após a cirurgia).

\subsection{Pinealectomia}

Para a retirada da pineal, os animais foram pesados, anestesiados com pentobarbital sódico (Nembutal, Abbot, $40 \mathrm{mg} / \mathrm{kg} / \mathrm{ip}$ ). Uma pequena incisão foi realizada na pele, no centro da região occipital. Após afastamento do seio sagital, a glândula pineal foi completamente removida por pinçamento, em sua haste, sendo procedida sutura dos planos cirúrgicos. Os animais receberam, profilaticamente, injeção de antibiótico (pentabiótico, Fontoura Wyeth, $0,4 \mathrm{ml} /$ animal $/ \mathrm{im}$ ).

A cirurgia fictícia foi realizada, seguindo-se as mesmas etapas da pinealectomia, inclusive o procedimento profilático com antibiótico; contudo, não houve retirada da glândula pineal.

\subsection{Coleta do Sangue e das Adrenais}

Sob anestesia inalatória com éter, abriu-se a caixa torácica dos animais, sendo feita punção do ven- trículo esquerdo com uso de seringa, contendo anticoagulante (heparina sódica; Eurofarma) e agulha hipodérmica $25 \times 7 \mathrm{~mm}$, para coleta de 3 a $5 \mathrm{ml}$ de sangue. Após a coleta do sangue, realizou-se perfusão no animal, utilizando-se o mesmo orifício criado na punção cardíaca. A perfusão foi iniciada com solução salina fisiológica $(\mathrm{NaCl} 0,9 \%)$ até o clareamento dos órgãos e, a seguir, utilizou-se formalina tamponada a $3,7 \%$ pH 7,4 até o enrijecimento do animal. Após a perfusão e morte do animal, foram feitas ressecção e pesagem das adrenais.

\subsection{Processamento Histológico das Adrenais}

Para a análise morfométrica e de proliferação celular das zonas corticais, as adrenais, após sua coleta, foram submetidas ao processamento histológico rotineiro, para preparação das lâminas, emblocadas em parafina e cortadas em micrótomo, com cortes em espessura de $5 \square \mathrm{m}$. As secções obtidas foram secadas em estufa a $37^{\circ} \mathrm{C}$ e coradas pelo método da hematoxilinaeosina, para a análise morfométrica, e imunoistoquímica, para a pesquisa da proliferação celular.

\subsection{Análise Morfométrica}

As lâminas contendo os fragmentos das adrenais foram avaliadas quanto à espessura de cada zona da cortical da adrenal (glomerular, fasciculada e reticular).

Para a determinação da espessura de cada zona do córtex da adrenal, utilizou-se microscópio de luz comum com objetiva 10x e com a régua milimetrada, existente no charriô do microscópio, fez-se a medição, sendo os resultados obtidos, expressos em milímetros.

\subsection{Análise da Proliferação Celular}

As lâminas contendo os cortes dos segmentos das adrenais foram analisadas, ao microscópio de luz comum, objetiva $400 \mathrm{x}$, para avaliar a proliferação celular existente nas diferentes zonas do córtex dessas glândulas. Utilizou-se o anticorpo monoclonal contra uma parte recombinante do antígeno Ki-67 (MIB-1, DAKO, Glostrup, Dinamarca), a uma diluição de 1:10, através do método imunoistoquímico.

A avaliação da taxa de células MIB-1 positivas foi realizada em cortes de adrenal de cada animal.

\subsection{Análise Estatística}

Todos os resultados obtidos dos parâmetros morfológicos e morfométricos dos grupos P1, P2 e $\mathrm{CF}$ foram comparados aos do grupo-controle normal 
pela análise de variância. A normalidade dos dados e homogeneidade das variâncias foram verificadas a partir dos testes Kolmogoxov-Smirnov e Barttett, respectivamente.Para as variáveis normais e variâncias constantes foi aplicada a ANOVA paramétrica (teste t-Studart).No caso das variáveis não normais foi utilizada a ANOVA não paramétrica, como o teste de Kruskall Wallis, seguido do teste de $2^{\text {a }}$ Ordem de Dunn. Todos os testes foram considerados siginificativos, quando $\mathrm{p}<0,05$.

\section{RESULTADOS}

De acordo com a análise morfométrica, constatou-se que somente na zona fasciculada existe diferença significante entre os grupos (normal, P1, P2 e
CF) $(p=0,001)$ (Tabelas I, II, III, IV).Nas zonas glomerular e reticular, a variabilidade entre os grupos não foi significante ( $\mathrm{p}=0,5 \mathrm{ep}=0,17$, respectivamente) e, nas associações entre os grupos, em relação à zona fasciculada, houve diferença estatisticamente significante entre CF e P1, CF e P2, (N + CF) e P1 e $(\mathrm{N}+$ $\mathrm{CF})$ e $\mathrm{P} 2$, sendo que as demais associações não apresentaram variação significante. Em relação às zonas glomerular e reticular, a diferença não existe. Além do mais, não foi encontrada variação significante de todas as zonas conjuntamente entre os grupos (ver tabelas).

Em relação à análise de proliferação celular, não se observaram células em proliferação, ou seja, positivas para o marcador MIB-1, em qualquer das zonas do córtex da adrenal, em todos os grupos estudados.

\begin{tabular}{|lcccccccccc|}
\hline \multicolumn{7}{|l}{ Tabela I: Medidas da espessura das zonas corticais da adrenal em milímetros no grupo controle (N) } \\
\hline RatosZonas & $R 1$ & $R 2$ & $R 3$ & $R 4$ & $R 5$ & $R 6$ & $R 7$ & $R 8$ & $R 9$ & $R 10$ \\
\hline Glomerular & 0,1 & 0,1 & 0,1 & 0,1 & 0,1 & 0,1 & 0,1 & 0,2 & 0,7 & 0,3 \\
\hline Fascicular & 0,4 & 0,3 & 0,4 & 0,4 & 0,4 & 0,5 & 0,4 & 0,4 & 0,7 & 0,9 \\
\hline Reticular & 0,7 & 0,2 & 0,4 & 0,4 & 0,6 & 0,2 & 0,4 & 0,5 & 0,3 & 0,7 \\
\hline
\end{tabular}

Tabela II: Medidas da espessura das zonas corticais da adrenal em milímetros no grupo experimental avaliado entre 15 e 25 dias após a pinealectomia (P1).

\begin{tabular}{|c|c|c|c|c|c|c|c|c|c|c|}
\hline & R1 & $\mathrm{R} 2$ & R3 & $\mathrm{R} 4$ & $\mathrm{R} 5$ & $\mathrm{R} 6$ & $\mathrm{R} 7$ & $\mathrm{R} 8$ & $\mathrm{Rg}$ & R10 \\
\hline Glomerular & 0,1 & 0,1 & 0,2 & 0,3 & 0,2 & 0,1 & 0,1 & 0,1 & 0,2 & 0,1 \\
\hline F ascicular & 0,8 & 0,7 & 0,6 & 0,7 & 0,6 & 0,7 & 0,7 & 0,3 & 0,7 & 0,5 \\
\hline Reticular & 0,5 & 0,2 & 0,2 & 0,3 & 0,3 & 0,3 & 0,2 & 0,4 & 0,7 & 0,4 \\
\hline
\end{tabular}

Tabela III: Medidas da espessura das zonas corticais da adrenal em milímetros do grupo experimental avaliado entre 60 e 70 dias após a pinealectomia (P3)

\begin{tabular}{|lccccccccccc|}
\hline Ratoszonas & $R 1$ & $R 2$ & $R 3$ & $R 4$ & $R 5$ & $R 6$ & $R 7$ & $R 8$ & $R 9$ & $R 10$ \\
\hline Glomerular & 0,1 & 0,1 & 0,1 & 0,1 & 0,1 & 0,1 & 0,1 & 0,2 & 0,1 & 0,3 \\
\hline Fascicular & 0,4 & 0,3 & 0,4 & 0,4 & 0,4 & 0,5 & 0,4 & 0,4 & 0,7 & 0,9 \\
\hline Reticular & 0,7 & 0,2 & 0,4 & 0,4 & 0,6 & 0,2 & 0,4 & 0,5 & 0,3 & 0,7 \\
\hline
\end{tabular}

\begin{tabular}{|ccccccccccc|c|}
\hline \multicolumn{8}{|c|}{ Tabela IV: Medida das zonas corticais da adrenal em milímetros do grupo de cirurgia fictícia (CF) } \\
\hline Ratos Zonas & $R 1$ & $R 2$ & $R 3$ & $R 4$ & $R 5$ & $R 6$ & $R 7$ & $R 8$ & $R 9$ & $R 10$ \\
\hline Glomerular & 0,1 & 0,1 & 0,1 & 0,1 & 0,1 & 0,1 & 0,1 & 0,1 & 0,1 & 0,1 \\
\hline Fascicular & 0,5 & 0,5 & 0,5 & 0,6 & 0,4 & 0,5 & 0,4 & 0,6 & 0,5 & 0,6 \\
\hline Reticular & 0,5 & 0,6 & 0,4 & 0,3 & 0,5 & 0,5 & 0,6 & 0,2 & 0,4 & 0,5 \\
\hline
\end{tabular}




\section{DISCUSSÃO}

De acordo com os nossos achados, apenas a zona fasciculada sofre influência da glândula pineal, em vista de a região apresentar-se com volume maior nos ratos pinealectomizados, avaliados 15 a 25 dias após pinealectomia. Esses resultados estão de acordo com os trabalhos apresentados por Nir et al.,1971 ${ }^{(6)}$ e Kosykhv et al., $1987^{(5)}$, que demonstraram aumento significativo da massa da adrenal murina após período que variou de 30 a 60 dias após a pinealectomia. Além disso, Vaughan et al.,1972 ${ }^{(13)}$ observaram que a hipertrofia adrenal após a pinealectomia pode ser inibida por injeções de melatonina nos ratos, demostrando a influência da pineal sobre o córtex da adrenal, o que reforça os resultados do presente trabalho.
Por outro lado, no presente estudo, não foram encontradas alterações na proliferação celular em nenhuma das zonas corticais da adrenal em todos os grupos analisados, confirmando os achados de Zieleniewski et al.,1986 ${ }^{(7)}$, que detectaram, após a pinealectomia, decréscimo no índice de mitose das células corticais da adrenal em comparação ao do grupo-controle.

Portanto, após a análise criteriosa dos dados da literatura mundial com os resultados do presente estudo, e que demonstra alterações morfológicas no córtex adrenal após a pinealectomia, é possível reforçar a afirmação de que a glândula pineal exerce influência sobre as adrenais ${ }^{(13)}$. A ausência da pineal causa aumento do peso da adrenal por hipertrofia celular da zona fasciculada e não hiperplasia, em vista de ocorrer diminuição ou inibição da proliferação celular do córtex da adrenal ${ }^{(5,6,7,14)}$.

LIMA LMB; LIMA MA; RITA CVC; OLIVEIRA PF; REIS LC; BORGES MF \& SILVA GP. Pineal's influence upon the adrenal cortex. Medicina, Ribeirão Preto, 35: 36-40, jan./march 2002.

ABSTRACT: Several glands interfere in the production and secretion of adrenocortical hormones. It has been demonstrated, by several studies, the inhibitory effect of pineal on these hormones. Therefore, the pinealectomy increases adrenocortical levels of hormones and promotes morphologic changes in the adrenal, such as decrement of mitotic activity and cortical hypertrophy. The aim of this present study was to verify the influence of the pinealectomy of rats adrenal glands. Four animals groups were selected, a control group, one submitted to sham surgery and two pinealectomized groups, sacrificed in different periods of time. Morphometric and cellular proliferation analysis of the adrenal cortical layers were performed. The morphometric study demonstrated an increase in the fasciculate layer from pinealectomized animals, as compared to control and sham surgery rats. The results showed that pineal exerts influence upon the adrenals and the absence of the pineal promotes weight increase of the adrenal by cellular hypertrophy, but not hyperplasia of the fasciculate layer.

UNITERMS: Pineal Body. Adrenal Cortex. Cell Division. Hypertrophy. Morphology.

\section{REFERÊNCIAS BIBLIOGRÁFICAS}

1 - JAYATILAK PG; SHETHAR \& SHAH PG. The role of adrenal and pineal glands on the hypothalamo-pituitary axis in adult male rats. Indian J Physiol Pharmacol 23: 365-377, 1979.

2 - OGLE TF \& KITAY JI. Effects of pinealectomy on adrenal function in vivo and in vitro in female rats. Endocrinology 98:20-24, 1976.

3-KINSOG; WAHID AD \& SINGER B. Effect of chronic pinealectomy on adrenocortical hormone secretion rates in normal and hipertensive rats. Gen Comp Endocrinol 8:445454, 1967.
4 - DEMISCH L; DEMISCH K \& NICKELSEN TM. Influence of dexamethasone on nocturnal melatonin production in health adult subjects. J Pineal Res 5: 317-322, 1988.

5 - KOSYKHV.A; LASUKOVA TV \& KOZLOVA IG. Status of the glucocorticoid function of the adrenal glands of the rat after pinealectomy. Probl Endokrinol (Mosk) 33: 258-260, 1987.

6 - NIR I; SCHMIDT U; HIRSCHMANN N \& SULMAN F G. The effect of pinealictomy on rat plasma corticosterone levels under various conditions of light. Life Sci [I] 10:6 317-24, 1971.

7 - ZIELENIEWSKI; J NOWAKOWSKA - JANKIEWICZ B; JUSZCZAK $M$ \& KARASEK M. Influence of pinealectomy on the mitotic activity of regenerating adrenal cortex in rats. Cytobios 47: 85-88, 1986. 
8 - KACHI T; QUAY WB; BANERTI TK \& IMAGAWA T. Effects of pinealectomy on the mitotic activity of adrenomedullary chromaffin cells in relation to time of day. J Pineal Res 8:21-34, 1990.

9 - LOBO RA \& LIMAOS EA. Inflammatory response modulated by pinealectomy: effect of light. Braz J Med Biol Res 24:1159-1162, 1991.

10 - YAMASAKI K; MARUBAYASHI V; REIS AM \& COIMBRA CC Preferential saline or water intake by pinealectomizedadrenalectomized male rats. Braz J Med Biol Res 23: 11771180, 1990.

11 - MALENDOWICZ LK; MAJCHRZAK M \& NOWAK M. Estradiol and melatonin effects on adrenal cortex of ovarectomized and pinealectomized rats. Exp Clin Endocrinol 85: 276282, 1985.
12 - KINSON G A; SINGER B \& GRANT L. Adrenocortical hormone secretion at various time intervals after pinealectomy in the rat. Gen Comp Endocrinol 10: 317-323,1968.

13 - VAUGHAN MK; VAUGHAN G.M; REITER R.J \& BENSON B. Effect of melatonin and other pineal indoles on adrenal enlargement produced in male and female mice by pinealectomy, unilateral adrenalectomy, castration, and cold stress. Neuroendocrinology 10: 139-154,1972.

14 - SWERYNEK E; LEWINSKI A; CHOCZAG V; SZKUDLINSKI M \& SZYMCZY KIEWICZ P. Melatonin - induced suppression of the pinealectomy - stimulated rat adrenal cortex mitotic activity. Cytobios 65: 115-122, 1991.

Recebido para publicação em 10/05/2001

Aprovado para publicação em 25/02/2002 